\title{
Pyruvate dehydrogenase deficiency
}

\author{
G K Brown, L J Otero, M LeGris, R M Brown
}

Genetics Laboratory,

Department of

Biochemistry,

University of Oxford,

South Parks Road,

Oxford OX1 3QU, UK

G K Brown

L J Otero

M LeGris

R M Brown

Dr G K Brown.
Correspondence to

Pyruvate dehydrogenase (PDH) deficiency is one of the most common of the defined genetic defects of mitochondrial energy metabolism. It is also one of the few $\mathrm{X}$ linked diseases in which a high proportion of heterozygous females manifest severe symptoms and the pathogenesis is understood. PDH deficiency is an extremely heterogeneous condition, both in clinical presentation and the severity of the biochemical abnormality, with a clinical spectrum ranging from fatal lactic acidosis in the newborn period to a chronic neurodegenerative condition with gross structural abnormalities in the central nervous system. ${ }^{1-3}$ The chronic neurological form of PDH deficiency represents an excellent illustration of the principle that structural defects, and in particular developmental anomalies, do not preclude a diagnosis of metabolic disease.

The biochemical abnormalities reflect the role of the PDH complex in normal energy metabolism. The enzyme catalyses the conversion of pyruvate (derived mainly from glucose) to acetyl coenzyme A, a key regulatory step in the central pathways of energy metabolism in the mitochondrion (fig 1). PDH is a multienzyme complex composed of multiple copies of nine different gene products. ${ }^{4}$ Five of the subunits make up the three enzyme activities which are involved in the conversion of py-

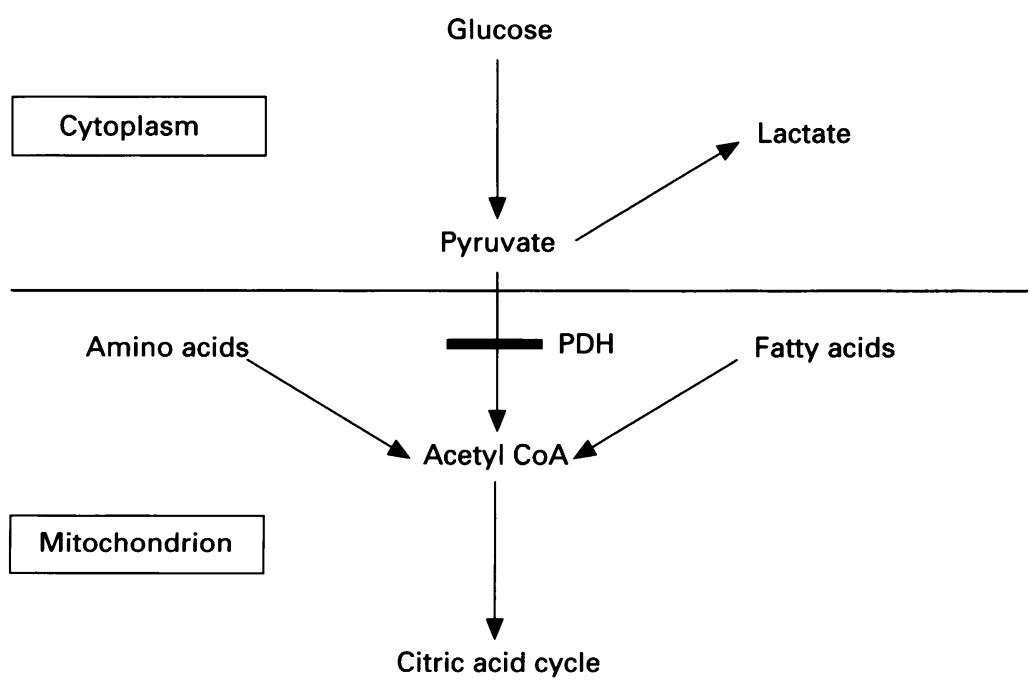

Figure 1 The role of the pyruvate dehydrogenase complex. The pyruvate dehydrogenase complex is a mitochondrial enzyme which converts pyruvate into acetyl CoA, thus linking the glycolytic pathway with the citric acid cycle. It is the point of entry of glucose into the central pathway of aerobic oxidation and is an important control point, determining the balance of energy generation from carbohydrates, fatty acids, and amino acids. ruvate to acetyl CoA. The others constitute specific regulatory components, a kinase and phosphatase, which control the activity of the entire complex (fig 2).

The level of activity of the PDH complex determines the balance between the use of alternative energy substrates and, in some tissues, the enzyme operates at near maximal rate. Consequently, relatively modest reductions in activity may lead to accumulation of pyruvate and, more importantly, lactic acid. Although the PDH complex plays an important role in all metabolically active tissues, it plays a critical role in the brain under normal conditions. This is because the brain usually obtains all of its energy from the aerobic oxidation of glucose. All patients with PDH deficiency have some degree of neurological impairment.

\section{Clinical presentations}

The following description of the features of $\mathrm{PDH}$ deficiency is based on our own experience of over 40 cases, and other published reports. Several hundred patients have now been described. PDH deficiency has a significant effect on fetal development and this may become apparent during late pregnancy with poor fetal weight gain and falling maternal urinary oestriol levels. Delivery may be complicated and the babies may have low Apgar scores. A low birth weight is very common. It has been suggested that there is a characteristic dysmorphic appearance associated with PDH deficiency, with a narrow head, frontal bossing, wide nasal bridge, long philtrum and flared nostrils, ${ }^{5}$ but this is not seen in all cases and is not specific to the disease. Other congenital malformations (simian creases, short neck, slight shortening of the limbs, flexion contractures, pes cavus, talipes, ventricular septal defect, and hydronephrosis) have been recorded in a few cases.

Patients with PDH deficiency typically develop symptoms soon after birth. In general, there are two forms of presentation, metabolic and neurological, and these occur at approximately equal frequency. ${ }^{6}$ The metabolic form presents as severe lactic acidosis with blood lactate concentration often $>10 \mathrm{mmol} / \mathrm{l}$. The lactic acidosis is almost always refractory to treatment and many of these patients die within the newborn period. In a very few cases, the lactic acidosis has been reported to respond to high doses of thiamine. ${ }^{7}$ A small number of patients with profound lactic acidosis have also 
E1

Figure 2 Molecular organisation of the pyruvate dehydrogenase complex. The conversion of pyruvate to acetyl $\mathrm{CoA}$ is catalysed by three enzyme activities. E1 (pyruvate dehydrogenase), E2 enzyme (dihydrolipoamide acetyltransferase), and E3 subunits. It removes the carboxyl group from pyruvate and transfers the remaining acetyl group to E2. This enzyme forms the structural core of the complex. It transfers the acetyl group to coenzyme $A$, generating acetyl $C o A$, the product of the overall reaction. In the course of this reaction, a lipoic acid cofactor on $E 2$ is reduced and this must be reoxidised for further cycles of activity. This reaction is catalysed by E3. The activity of the whole complex is regulated by phosphorylation. A specific PDH kinase phosphorylates specific serine residues in the E1 1 subunit, inactivating the whole complex. Reactivation is catalysed by a specific phosphatase.

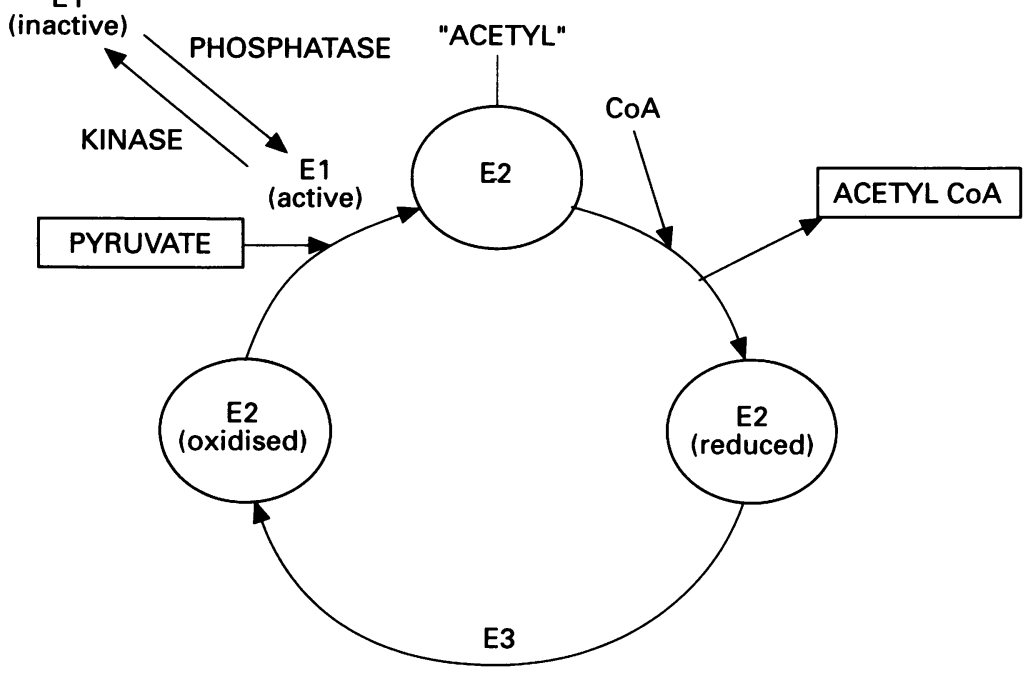
(dihydrolipoamide dehydrogenase). E1 is a tetrameric protein with two $\alpha$ and two $\beta$

gresses to profound mental retardation, microcephaly, blindness, and spasticity with secondary contractures. Long term survival is possible and several of these patients have reached their teens. ${ }^{10}$

Between these two extremes, there is a continuous spectrum of intermediate forms. When the metabolic abnormalities are less severe, the onset may be delayed until later in infancy and the clinical course is often characterised by intermittent episodes of lactic acidosis, often precipitated by intercurrent illness, and associated with cerebellar ataxia. ${ }^{5}$ A number of the patients with primarily neurological symptoms fit into the category of Leigh's syndrome, a subacute neurodegenerative disease with prominent brain stem features. ${ }^{11}$

Although PDH deficiency occurs with equal frequency in males and females, the presentation of the disease differs between the sexes. The metabolic end of the spectrum, especially the severe neonatal lactic acidosis, predominates in males and the chronic neurological form is much more common in females. Neurological disease in males often manifests as Leigh's syndrome. In patients with prominent neurological symptoms, there are often major structural defects in the brain (fig 3) and metabolic abnormalities may be absent or minimal. In these cases, a possible diagnosis of $\mathrm{PDH}$ deficiency may be overlooked.

\section{Neuropathology}

Structural abnormalities in the brain are one of the most striking aspects of PDH deficiency. The lesions are generated by two different pathological processes, degenerative change and developmental malformation, ${ }^{12}$ and are seen most clearly in patients with the chronic neurological form of the disease. These patients typically have marked cerebral atrophy and ventricular dilatation (fig 3), sometimes to the extent that a diagnosis of hydranencephaly is considered. In addition to the destructive changes, there is a characteristic pattern of developmental anomalies which includes complete or partial absence of the corpus callosum, absence of the medullary pyramids, and abnormal and ectopic inferior olives. Patients presenting as Leigh's syndrome have the characteristic pathological changes of symmetrical cystic lesions and gliosis in the basal ganglia and brain stem, accompanied by capillary proliferation. ${ }^{13}$ Surprisingly, patients with early onset severe lactic acidosis, particularly males, may have remarkably little neuropathology. ${ }^{14}$ In some there is generalised hypomyelination, others have small cystic lesions and gliosis in the cortex, basal ganglia, brain stem, or cerebellum.

Figure 3 Magnetic resonance image of the brain of a female patient. A magnetic resonance image of a female patient with a defined mutation in the E1 $\alpha$ subunit gene showing typical extensive cerebral atrophy and ventricular dilatation.

had significant hyperammonaemia in the newborn period. ${ }^{89}$ Patients with neurological presentation are hypotonic, feed poorly, and are lethargic. Later, they develop seizures, either tonic/clonic convulsions or typical infantile spasms. This form of the disease usually pro-

\section{Diagnosis}

The diagnosis of PDH deficiency should be considered in all patients with early onset neurological disease, especially if associated with structural abnormalities in the brain and a lactic acidosis for which there is no other apparent cause. Many of the neuropathological changes may be detected by ultrasound in the 
newborn period or with CT or MRI scans of the brain. When the blood lactate is not significantly raised, an important clue to the diagnosis is selective elevation of the lactate concentration in cerebrospinal fluid. ${ }^{10}$ If proton magnetic resonance spectroscopy of the brain is available, it can be used to show accumulation of lactate in the central nervous system. ${ }^{15}$

The differential diagnosis of primary lactic acidosis includes other defects of pyruvate metabolism (such as pyruvate carboxylase deficiency), defects of gluconeogenesis, and disorders of the mitochondrial electron transport chain. Isolated accumulation of lactic acid is the common metabolic abnormality in all of these conditions, although hypoglycaemia, especially after fasting, may be a prominent feature of the defects of gluconeogenesis. Early onset mitochondrial disease often involves multiple tissues including muscle, heart, liver, and kidney. These organs are not involved in PDH deficiency as they are able to use substrates other than glucose for energy. In all of these conditions, the diagnosis is based on specific biochemical investigations.

Specific enzyme assays have been designed which allow the measurement both of the overall maximal PDH activity and that of the individual components of the multi-subunit complex. The great majority of patients suffering from PDH deficiency are found to be defective in the $\mathrm{E} 1$ enzyme but abnormalities have also been detected in the E2 and E3 components. ${ }^{1316}$ Results of these assays show a wide range in residual enzyme activity and a poor correlation with clinical presentation, although, in general, patients with lower residual activity present earlier and with more severe symptoms. Further diagnostic information may be obtained from immunochemical analysis. ${ }^{17}$ However, a wide variation in the levels of immunological cross reacting material is observed which, again, does not correlate well with the levels of residual enzyme activity. For example, it is possible to find normal amounts of cross reacting material in patients who are severely deficient in PDH activity.

Elucidation of the genetic basis of PDH deficiency has partly explained the extensive biochemical and immunochemical heterogeneity of this condition. In almost all cases, a defect in the gene for the E1 $\alpha$ subunit is responsible. The gene for this subunit has been cloned and localised to Xp22.1. ${ }^{18}$ The X chromosome location of the PDH E1 $\alpha$ gene partly accounts for the different patterns of presentation of PDH deficiency observed between males and females. In males, all cells express the defective enzyme and the severe metabolic presentation is common. In female patients, the normal allele is expressed in a proportion of the cells, depending on the pattern of $X$ chromosome inactivation, and this may supply sufficient active enzyme to fulfil the needs of most tissues. The threshold for abnormal function in heterozygous females will differ in different tissues, depending on their energy requirements and their ability to use alternative energy substrates, such as fatty acids or amino acids. It appears that this threshold is exceeded in the brain in the majority of heterozygous females as they commonly manifest neurological symptoms, although they are often spared the systemic metabolic acidosis which is so prominent in males.

Cloning of the PDH E1 $\alpha$ gene has allowed the identification of the underlying mutation in a number of individual patients. ${ }^{10}$ The majority of these are new mutations, although a small number have been described in more than one patient. The wide range of enzyme activities seen presumably reflects the differential effects of specific mutations on the expression, stability, and activity of the mutant protein. A further implication of this mutational heterogeneity is that it is not possible to set up a routine diagnostic service based on screening for one or two common mutations. For diagnosis at the molecular genetic level, it is necessary to examine each patient individually. Previous knowledge of a mutation may assist in prenatal diagnosis for parents who have already borne affected offspring if, for example, the mutation is such that it allows simple detection by generation or removal of a restriction site.

\section{PDH E1 $\alpha$ deficiency in heterozygous females}

Almost all females known to be heterozygous for PDH E1 $\alpha$ mutations have manifested the disease and most have died before reaching adulthood. However, the equal number of affected males and females indicates that PDH $\mathrm{E} 1 \alpha$ deficiency is not a fully dominant disease and it is therefore likely that there will be some normal or very mildly affected females. Normally, these will only be detected if they give birth to a more severely affected child and, indeed, a few such cases have been identified. ${ }^{20}$ The manifestations of the disease in these females have been restricted to seizures and a variable degree of mental retardation. In one case, the brain appeared normal on CT $\operatorname{scan}^{20}$ and imaging studies are not indicated as a routine investigation of the mothers of severely affected patients. It is not known if adult females with mild neurological symptoms have consistently raised lactate concentration in cerebrospinal fluid. The striking difference in clinical presentation of females possessing the same mutation can be accounted for by the complicating factor of $\mathrm{X}$ inactivation.

In fact, variations in $\mathrm{X}$ inactivation patterns appear to be responsible for much of the wide variation in clinical severity in heterozygous females, as mutation analysis has shown that the majority of these females have functionally equivalent frameshift mutations which result in complete deficiency of PDH E1 $\alpha$ protein. As a consequence of random $\mathrm{X}$ inactivation, some cells will express the $\mathrm{X}$ chromosome carrying the normal PDH El $\alpha$ gene and others will express the $\mathrm{X}$ chromosome carrying the mutation. This mosaicism can be shown in cultured fibroblasts from patients with null mutations by immunochemical staining with specific $\mathrm{E} 1 \alpha$ antibodies. ${ }^{18}$ The proportion of 
cells expressing the normal gene may vary considerably, both in different tissues of the same person ${ }^{21}$ and between different persons. ${ }^{20} \mathrm{Com}-$ plete PDH deficiency has not been identified in males and present evidence suggests that it is incompatible with fetal development. In heterozygous females, even a relatively small population of cells expressing the normal PDH $\mathrm{E} 1 \propto$ gene appears to be sufficient to allow survival, at least until birth.

Females present a special problem in diagnosis of PDH deficiency when this is based on measurement of enzyme activity. When the proportion of cells expressing the normal $\mathrm{X}$ chromosome exceeds $\sim 75 \%$, enzyme activity and levels of immunoreactive protein cannot be distinguished from the range in normal controls and the diagnosis may be missed. In cultured fibroblasts, which are commonly used for diagnosis, an $\mathrm{X}$ inactivation ratio of $>3: 1$ is found in approximately $25 \%$ of cultures ${ }^{22}$ and in these cases the diagnosis is not excluded by normal enzyme assay results. When the clinical and biochemical features strongly suggest PDH deficiency and the fibroblast enzyme activity is in the normal range, a skewed $\mathrm{X}$ inactivation pattern of this order makes it mandatory to screen the PDH E1 $\alpha$ gene for a mutation. The diagnosis of PDH E1 $\alpha$ deficiency has been established in this way in a number of patients who were initially assessed as normal by enzyme assay. $^{23}$

\section{Prenatal diagnosis}

PDH deficiency is a severe disease which results in the death of the great majority of patients before they reach adulthood. Consequently, most of the cases are sporadic and result from a new mutation arising within the germ cells of one of the parents. Although the recurrence risk for PDH E1 $\alpha$ deficiency is usually very low, the occurrence of a small number of families with affected sibs, the severity of the disease, and the possibility of germline mosaicism has meant that prenatal diagnosis is almost always requested after diagnosis of an affected child. If the diagnosis is to be based on measurement of enzyme activity, cultured chorionic villus cells are the most suitable fetal material. Repeat assays on living cells, grown under standardised conditions, are needed for reliable diagnosis and chorionic villus cell culture is the optimal means of ensuring that this is achieved before the pregnancy is too advanced. If amniotic cells are used, it usually takes three to four weeks of cell culture before there is sufficient material for the assays.

When the fetus is male, or the enzyme activity in chorionic villus cells is significantly reduced, the diagnosis can be made with confidence. However, extreme skewing of $\mathrm{X}$ inactivation patterns presents a major problem when prenatal diagnosis of PDH E1 $\alpha$ deficiency in female fetuses is based on enzyme activity measurements in cultured chorionic villus cells. Our experience with chorionic villus cell cultures indicates that not only are many of these clonal, but there may also be a poor correlation between $\mathrm{X}$ inactivation and the methylation status at hypervariable $\mathrm{X}$ linked loci because the adult pattern of methylation has not been established in these cells. ${ }^{22}$ Unless the parental origin of the mutant chromosome in a previously affected child has been determined and it is certain which $\mathrm{X}$ chromosome is active in the chorionic villus cells, the diagnosis is not excluded when there is normal enzyme activity and a skewed $\mathrm{X}$ inactivation pattern. Clearly, identification of the mutation in an affected child and direct genetic analysis in subsequent pregnancies is the only completely reliable method of prenatal diagnosis. ${ }^{24}$

\section{Treatment}

Treatment of PDH deficiency rarely influences the course of the disease. It is usually possible to reverse or minimise systemic lactic acid accumulation by giving a high fat/low carbohydrate "ketogenic" diet, but this does not alleviate the neurological symptoms as structural damage in the brain is present from before birth and many patients do not have significant metabolic problems. There is some evidence that dichloroacetate (which inhibits the specific PDH kinase and thereby activates any residual functioning complex) will also reduce the metabolic disturbance in some patients, but, again, this is rarely accompanied by any objective improvement in neurological performance. A more favourable outcome can be expected in the extremely rare patients with a thiamine responsive form of the disease and, for this reason, a short therapeutic trial of thiamine is worth trying in all cases.

PDH deficiency is a clinically heterogeneous neurometabolic disease in which biochemical abnormalities may be minimal and easily overlooked, especially when the patient has extensive structural defects in the brain. The most common genetic cause of PDH deficiency is mutation in the gene for the $\mathrm{E} 1 \alpha$ subunit. PDH $\mathrm{E} 1 \alpha$ deficiency is an unusual $\mathrm{X}$ linked disorder in which males and females are equally affected and most cases are sporadic. Variable manifestation of the disease in heterozygous females appears to be determined largely by differences in the pattern of $\mathrm{X}$ inactivation and this also results in considerable difficulties in establishing the diagnosis when this is based on measurements of enzyme activity and immunoreactive protein.

We wish to thank Mrs Nada Al Murani for assistance with the patients' biochemical studies and Dr J H Cross, Institute of patients' biochemical studies and Dr f H Cross, Institute of child Health, University of London, for providing the clinical photograph. This work was supporte

1 Robinson BH, Chun K, Mackay N, Otulakowski G, PetrovaBenedict $R$, Willard $\mathrm{H}$. Isolated and combined deficiencies Benedict $\mathrm{R}$, Willard $\mathrm{H}$. Isolated and combined deficiencies of the $\alpha$-keto acid dehydro
Acad Sci 1989;573:337-46.

2 Ho L, Wexler ID, Kerr DS, Patel MS. Genetic defects in human pyruvate dehydrogenase. Ann N Y Acad Sci 1989; human pyru.

3 Brown GK, Brown RM, Scholem RD, Kirby DM, Dahl HHM. The clinical and biochemical spectrum of human HHM. The clinical and biochemical spectrum of human pyruvate dehydroge
$1989 ; 573: 360-8$

4 Patel MS, Roche TE. Molecular biology and biochemistry of pyruvate dehydrogenase complexes. FASEB $\mathcal{f} 1990$; 432:24-33. 
5 Robinson BH, MacMillan H, Petrova-Benedict $R$, Sherwood WG. Variable clinical presentation in patients with defective E1 component of pyruvate patients with defective E1 component of pyru
dehydrogenase complex. $\mathcal{F}$ Pediatr 1987;111:525-33.

6 Brown GK. Pyruvate dehydrogenase E1 $\alpha$ deficiency. $f$ Inherited Metab Dis 1992;15:625-33.

7 Narisawa K, Endo H, Miyabayashi S, Tada K. Thiamine responsive pyruvate dehydrogenase deficiency. $\mathcal{F} \mathrm{Nutr} S c i$ Vitaminol Tokyo 1992;5:85-8.

8 Byrd DJ, Krohn HP, Winkler L, et al. Neonatal pyruvate dehydrogenase deficiency with lipoate responsive lactic acidaemia and hyperammonaemia. Eur $f$ Pediatr 1989; 148:543-7.

9 Brown GK, Scholem RD, Hunt SM, Harrison JR, Pollard AC. Hyperammonaemia and lactic acidosis in a patient with pyruvate dehydrogenase deficiency. $\mathcal{F}$ Inherited Metab Dis 1987;10:359-66.

10 Brown GK, Haan EA, Kirby DM, et al. "Cerebral" lactic acidosis: defects in pyruvate metabolism with profound brain damage and minimal systemic acidosis. Eur $¥$ Pediatr brain damage and

11 Kretzschmar HA, DeArmond SJ, Koch TK, et al. Pyruvate dehydrogenase complex deficiency as a cause of subacute necrotizing encephalopathy (Leigh disease). Pediatrics 1987;79:370-3.

12 Chow CW, Anderson RM, Kenny GCT. Neuropathology in cerebral lactic acidosis. Acta Neuropathol (Berl) 1987; 74:393-6.

13 Montpetit VJA, Andermann F, Carpenter S, Fawcett JS. Zbrowska-Sluis D, Giberson HR. Subacute necrotizing encephalomyelopathy: a review and a study of two families. Brain 1971;94:1-30.

14 Hansen LL, Brown GK, Kirby DM, Dahl HHM. Characterization of the mutations in three patients with pyruvate dehydrogenase $\mathrm{E} 1 \alpha$ deficiency. $\mathcal{F}$ Inherited Metab Dis 1991;14:140-51.

15 Cross JH, Connelly A, Gadian DG, et al. The clinical diversity of pyruvate dehydrogenase deficiency. Pediatr Neurol 1994;10:276-83.

16 Robinson BH, MacKay N, Petrova-Benedict R, Ozalp I, Coskun T, Stackpoole PW. Defects in the E2 lipoyl transacetylase and the X-lipoyl containing component of the pyruvate dehydrogenase complex in patients with lactic acidemia. $\mathcal{f}$ Clin Invest $1990 ; 85: 1821-4$.

17 Wicking CA, Scholem RD, Hunt SM, Brown GK. Immunochemical analysis of normal and mutant forms of human pyruvate dehydrogenase. Biochem $\mathcal{f}$ 1986;239:8996.

18 Brown RM, Dahl HHM, Brown GK. X-chromosome localization of the functional gene for the E1 $\alpha$ subunit of the human pyruvate dehydrogenase complex. Genomics 1989 ; 41:74-81.

19 Dahl HHM, Brown GK, Brown RM, et al. Mutations and polymorphisms in the pyruvate dehydrogenase E1 $\alpha$ gene. Hum Mutat 1992;1:97-102.

20 Dahl HHM, Hansen LL, Brown RM, Danks DM, Rogers JG, Brown GK. X-linked pyruvate dehydrogenase E1 $\alpha$ subunit deficiency in heterozygous females: variable masubunit deficiency in heterozygous females: variable ma1992;15:835-47.

21 Brown RM, Fraser NJ, Brown GK. Differential methylation of the hypervariable locus DXS255 on active and inactive $\mathrm{X}$ chromosomes correlates with the expression of a human X-linked gene. Genomics 1990;7:215-21

22 Brown RM, Brown GK. X chromosome inactivation and the diagnosis of $\mathrm{X}$ linked disease in females. $\mathcal{F}$ Med Genet 1993;30:177-84.

23 Matthews PM, Brown RM, Otero LJ, et al. Pyruvate dehydrogenase deficiency: clinical presentation and molecular genetic characterisation of five new patients. Brain 1994;117:435-43.

24 Brown RM, Brown GK. Prenatal diagnosis of pyruvate dehydrogenase E1 $\alpha$ subunit deficiency. Prenat Diagn 1994;14: 435-42. 